\title{
High Accuracy Ellipse-Specific Fitting
}

\author{
Tomonari Masuzaki ${ }^{1, \star}$, Yasuyuki Sugaya ${ }^{1}$, and Kenichi Kanatani ${ }^{2}$ \\ 1 Toyohashi University of Technology \\ \{masuzaki, sugaya\}@iim.cs.tut.ac.jp \\ 2 Okayama University \\ kanatani2013@yahoo.co.jp
}

\begin{abstract}
We propose a new method that always fits an ellipse to a point sequence extracted from images. The currently known best ellipse fitting method is hyper-renormalization of Kanatani et al., but it may return a hyperbola when the noise in the data is very large. Our proposed method returns an ellipse close to the point sequence by random sampling of data points. Doing simulation, we show that our method has higher accuracy than the method of Fitzgibbon et al. and the method of Szpak et al., the two methods so far proposed to always return an ellipse.
\end{abstract}

Keywords: ellipse-specific fitting, random sampling, hyperaccuracy correction.

\section{Introduction}

Detecting circles and ellipses in images is the first step of many computer vision applications including industrial robotic operations and autonomous navigation 468. To this end, point sequences constituting elliptic arcs are detected by image processing operations [51120 21/22], and then an ellipse equation is fitted to them. The simplest technique for the latter is to minimize the algebraic distance, i.e., the sum of squares of the ellipse equation. The solution is obtained analytically. Since the algebraic distance is 0 if all coefficients are 0 , we need to impose some constraint on them, and the solution depends on it. Methods of this type are called algebraic. The oldest and best known algebraic method is least squares $(L S)$, which constrains the sum of squares of the coefficients to 1. A more accurate algebraic method was proposed by Taubin [24]. The most accurate algebraic method known is HyperLS [1314].

Although algebraic methods do not require iterations, we can achieve higher accuracy by incorporating iterations. The standard procedure is maximum likelihood $(M L)$, which minimizes the reprojection error, i.e., the sum of the distances from data points to the fitted ellipse [4. The reprojection error is well approximated by what is known as the Sampson error 4], which can be minimized by various means including the FNS (Fundamental Numerical Scheme) of Chojnatcki et al. 2], the HEIV (Heteroscedastic Errors-in-Variable) of Leedan and

\footnotetext{
* Corresponding author.
} 


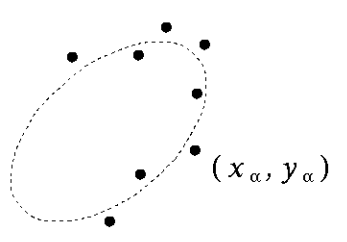

Fig. 1. Fit an ellipse to given points

Meer 18 and Matei and Leedan [19, and the projective Gauss-Newton iterations of Kanatani and Sugaya [15. The exact ML solution can be obtained by iterating Sampson error minimization [16.

Rather than minimizing some cost function, we may directly derive equations to solve so that the resulting solution has high accuracy. The oldest method of this type is iterative reweight, which was improved by Kanatani [7/8] to a high accuracy scheme called renormalization. It was further improved by AlSharadqah and Chernov [1] and Kanatani et al. 12] to hyper-renormalization. On the other hand, one can apply what is called hyperaccurate correction to the ML solution so as to further improve the accuracy 9 17. Hyper-renormalization and hyperaccurate correction both achieve the theoretical accuracy limit called the $K C R$ lower bound 810 up to a high degree and are regarded as the most accurate of all currently known methods.

However, all these are methods to fit a quadratic equation in $x$ and $y$, or a conic, to a point sequence. Usually, an ellipse results if the sequence is extracted from an elliptic arc, but a hyperbola or a parabola could result when the sequence is very short and the noise is very large. It is Fitzgibbon et al. 3] who first proposed a method that only fits an ellipse. It is an algebraic method, and the computation is very easy, but the accuracy is low. Recently, Szpak et al. 23. introduced a high accuracy ellipse-specific method based on Sampson error minimization. In this paper, we incorporate to hyper-renormalization a procedure for avoiding non-ellipses and demonstrate by experiments that our technique outperforms the method of Szpak et al. 23.

\section{Ellipse Fitting}

Curves represented by a quadratic equations in $x$ and $y$ in the form

$$
A x^{2}+2 B x y+C y^{2}+2 f_{0}(D x+E y)+f_{0}^{2} F=0,
$$

are called conics, which include ellipses, parabolas, hyperbolas, and their degeneracies such as two lines 6. The condition that Eq. (1) represents an ellipse is

$$
A C-B^{2}>0 \text {. }
$$

Our task is to compute the coefficients $A, \ldots, F$ so that the ellipse of Eq. (11) passes through given points $\left(x_{\alpha}, y_{\alpha}\right), \alpha=1, \ldots, N$, as closely possible (Fig. 1). In 
Eq. (11), $f_{0}$ is a constant that has the order of the image size for stabilizing finite length numerical computation (we set $f_{0}=600$ in our experiments). For a point sequence $\left(x_{\alpha}, y_{\alpha}\right), \alpha=1, \ldots, N$, we define 6 -D vectors

$$
\begin{aligned}
\boldsymbol{\xi}_{\alpha} & =\left(x_{\alpha}^{2}, 2 x_{\alpha} y_{\alpha}, y_{\alpha}^{2}, 2 f_{0} x_{\alpha}, 2 f_{0} y_{\alpha}, f_{0}^{2}\right)^{\top}, \\
\boldsymbol{\theta} & =(A, B, C, D, E, F)^{\top} .
\end{aligned}
$$

The condition that $\left(x_{\alpha}, y_{\alpha}\right)$ satisfies Eq. (10) is written as

$$
\left(\boldsymbol{\xi}_{\alpha}, \boldsymbol{\theta}\right)=0,
$$

where $(\boldsymbol{a}, \boldsymbol{b})$ denotes the inner product of vectors $\boldsymbol{a}$ and $\boldsymbol{b}$. Since vector $\boldsymbol{\theta}$ has scale indeterminacy, we normalize it to unit norm: $\|\boldsymbol{\theta}\|=1$.

Since Eq. (4) is not exactly satisfied in the presence of noise, we compute a $\boldsymbol{\theta}$ such that $\left(\boldsymbol{\xi}_{\alpha}, \boldsymbol{\theta}\right) \approx 0, \alpha=1, \ldots, N$. For computing a $\boldsymbol{\theta}$ that is close to its true value, we need to consider the statistical properties of noise. The standard model is to regard the noise in $\left(x_{\alpha}, y_{\alpha}\right)$ as independent Gaussian random variable of mean 0 and standard deviation $\sigma$. Then, the covariance matrix of the vector $\boldsymbol{\xi}_{\alpha}$ has the form $\sigma^{2} V_{0}\left[\boldsymbol{\xi}_{\alpha}\right]$, where

$$
V_{0}\left[\boldsymbol{\xi}_{\alpha}\right]=4\left(\begin{array}{cccccc}
x_{\alpha}^{2} & x_{\alpha} y_{\alpha} & 0 & f_{0} x_{\alpha} & 0 & 0 \\
x_{\alpha} y_{\alpha} & x_{\alpha}^{2}+y_{\alpha}^{2} & x_{\alpha} y_{\alpha} & f_{0} y_{\alpha} & f_{0} x_{\alpha} & 0 \\
0 & x_{\alpha} y_{\alpha} & y_{\alpha}^{2} & 0 & f_{0} y_{\alpha} & 0 \\
f_{0} x_{\alpha} & f_{0} y_{\alpha} & 0 & f_{0}^{2} & 0 & 0 \\
0 & f_{0} x_{\alpha} & f_{0} y_{\alpha} & 0 & f_{0}^{2} & 0 \\
0 & 0 & 0 & 0 & 0 & 0
\end{array}\right),
$$

which we call the normalized covariance matrix [8]10|15].

\section{Hyper-renormalization}

The hyper-renormalization of Kanatani et al. 12 can be described as follows:

1. Let $W_{\alpha}=1, \alpha=1, \ldots, N$, and $\boldsymbol{\theta}_{0}=\mathbf{0}$.

2. Compute the following matrices $\boldsymbol{M}$ and $\boldsymbol{N}$ :

$$
\begin{aligned}
\boldsymbol{M}= & \frac{1}{N} \sum_{\alpha=1}^{N} W_{\alpha} \boldsymbol{\xi}_{\alpha} \boldsymbol{\xi}_{\alpha}^{\top}, \\
\boldsymbol{N}= & \frac{1}{N} \sum_{\alpha=1}^{N} W_{\alpha}\left(V_{0}\left[\boldsymbol{\xi}_{\alpha}\right]+2 \mathcal{S}\left[\boldsymbol{\xi}_{\alpha} \boldsymbol{e}^{\top}\right]\right) \\
& -\frac{1}{N^{2}} \sum_{\alpha=1}^{N} W_{\alpha}^{2}\left(\left(\boldsymbol{\xi}_{\alpha}, \boldsymbol{M}_{5}^{-} \boldsymbol{\xi}_{\alpha}\right) V_{0}\left[\boldsymbol{\xi}_{\alpha}\right]+2 \mathcal{S}\left[V_{0}\left[\boldsymbol{\xi}_{\alpha}\right] \boldsymbol{M}_{5}^{-} \boldsymbol{\xi}_{\alpha} \boldsymbol{\xi}_{\alpha}^{\top}\right]\right),
\end{aligned}
$$

where $\mathcal{S}[\cdot]$ denotes symmetrization $\left(\mathcal{S}[\boldsymbol{A}]=\left(\boldsymbol{A}+\boldsymbol{A}^{\top}\right) / 2\right)$ and the vector $\boldsymbol{e}$ is defined to be

$$
\boldsymbol{e}=(1,0,1,0,0,0)^{\top} .
$$

The symbol $\boldsymbol{M}_{5}^{-}$denotes the pseudoinverse of $\boldsymbol{M}$ with truncated rank 5 , i.e., with the smallest eigenvalue replaced by 0 in the spectral decomposition. 
3. Solve the generalized eigenvalue problem

$$
\boldsymbol{N} \boldsymbol{\theta}=\mu \boldsymbol{M \theta},
$$

and compute the unit eigenvector $\boldsymbol{\theta}$ for the largest eigenvalue $\mu$.

4. If $\boldsymbol{\theta} \approx \boldsymbol{\theta}_{0}$ up to sign, return $\boldsymbol{\theta}$ and stop. Else, let

$$
W_{\alpha} \leftarrow \frac{1}{\left(\boldsymbol{\theta}, V_{0}\left[\boldsymbol{\xi}_{\alpha}\right] \boldsymbol{\theta}\right)}, \quad \boldsymbol{\theta}_{0} \leftarrow \boldsymbol{\theta},
$$

and go back to Step 2.

Hyper-renormalization is regarded as achieving practically the highest accuracy among existing methods, but it returns a non-ellipse, typically a hyperbola [12, when the sequence is very short and the noise is very large, as our experiments later show.

\section{Ellipse Specific Methods}

For fitting only an ellipse, Fitzgibbon et al. 3] proposed to minimize the algebraic distance

$$
J_{\mathrm{LS}}=\sum_{\alpha=1}^{N}\left(\boldsymbol{\xi}_{\alpha}, \boldsymbol{\theta}\right)^{2},
$$

subject to $A C-B^{2}=1$. This constraint is written as

$$
\left(\boldsymbol{\theta}, \boldsymbol{N}_{\mathrm{F}} \boldsymbol{\theta}\right)=1, \quad \boldsymbol{N}_{\mathrm{F}} \equiv\left(\begin{array}{rrrrrr}
0 & 0 & 1 & 0 & 0 & 0 \\
0 & -2 & 0 & 0 & 0 & 0 \\
1 & 0 & 0 & 0 & 0 & 0 \\
0 & 0 & 0 & 0 & 0 & 0 \\
0 & 0 & 0 & 0 & 0 & 0 \\
0 & 0 & 0 & 0 & 0 & 0
\end{array}\right)
$$

The solution that minimizes Eq. (10) subject to this constraint is obtained by solving a generalized eigenvalue problem

$$
\boldsymbol{N}_{\mathrm{F}} \boldsymbol{\theta}=\mu \boldsymbol{M}_{\mathrm{LS}} \boldsymbol{\theta}
$$

and computing the unit eigenvector $\boldsymbol{\theta}$ for the largest eigenvalue $\mu$, where the matrix $M_{\mathrm{LS}}$ is defined by

$$
\boldsymbol{M}_{\mathrm{LS}}=\frac{1}{N} \sum_{\alpha=1}^{N} \boldsymbol{\xi}_{\alpha} \boldsymbol{\xi}_{\alpha}^{\top}
$$

It has been widely observed, however, that the resulting ellipse is heavily biased. This is because the algebraic distance does not take into account the statistical properties of $\boldsymbol{\xi}_{\alpha}$ expressed by its covariance matrix. 
Recently, Szpak et al. 23] proposed a new ellipse specific method. They minimized

$$
J=\frac{1}{N} \sum_{\alpha=1}^{N} \frac{\left(\boldsymbol{\xi}_{\alpha}, \boldsymbol{\theta}\right)^{2}}{\left(\boldsymbol{\theta}, V_{0}\left[\boldsymbol{\xi}_{\alpha}\right] \boldsymbol{\theta}\right)}+\frac{\lambda\|\boldsymbol{\theta}\|^{4}}{(\boldsymbol{\theta}, \boldsymbol{N} \boldsymbol{\theta})^{2}}
$$

where $\lambda$ is a small constant. The first term on the right is called the Sampson error, which approximates the reprojection error up to high order noise terms. The second term on the right diverges if $\left(\boldsymbol{\theta}, \boldsymbol{N}_{\mathrm{F}} \boldsymbol{\theta}\right)=0$, i.e., if $\boldsymbol{\theta}$ represents a parabola. Since the domain of $\boldsymbol{\theta}$ is separated into the region $\left(\boldsymbol{\theta}, \boldsymbol{N}_{\mathrm{F}} \boldsymbol{\theta}\right)>0$ of ellipses and the region $\left(\boldsymbol{\theta}, \boldsymbol{N}_{\mathrm{F}} \boldsymbol{\theta}\right)<0$ of hyperbolas, minimization search starting from the ellipse domain does not cross the boundary $\left(\boldsymbol{\theta}, \boldsymbol{N}_{\mathrm{F}} \boldsymbol{\theta}\right)=0$, on which $J$ is $\infty$. Szpak et al. 23] used the Levenberg-Marquardt method for minimizing Eq. (14), choosing $\lambda$ as small as possible as long as an ellipse results.

\section{Proposed Method}

It has been observed that the accuracy of hyper-renormalization is higher than Sampson error minimization [1217]. Hence, it is reasonable to retain the solution of hyper-renormalization as long as it is an ellipse. If the hyper-renormalization iterations do not converge within a fixed limit, or if the resulting solution is not an ellipse, we switch to random sampling: we randomly choose from the point sequence five different points and compute the conic that passes through them. If a non-ellipse results, we discard the five points and choose new five points. If an ellipse results, we compute its Sampson error (the first term on the right of Eq. (14)) and choose the solution for which the Sampson error is the smallest. The procedure is summarized as follows:

1. Fit an ellipse to a point sequence $\left(x_{\alpha}, y_{\alpha}\right), \alpha=1, \ldots, N$, by hyperrenormalization, and compute its parameter vector $\boldsymbol{\theta}$. If

$$
\left(\boldsymbol{\theta}, \boldsymbol{N}_{\mathrm{F}} \boldsymbol{\theta}\right)>0,
$$

return $\boldsymbol{\theta}$ and stop.

2. If the hyper-renormalization iterations do not converge within a fixed limit (we set it to 100 times in our experiment), or if the resulting solution is not satisfy Eq. (15), we randomly choose five different points and compute the conic that passes through them.

3. If $\boldsymbol{\theta}$ does not satisfy Eq. (15), we discard the five points and choose new five points.

4. If the computed $\boldsymbol{\theta}$ satisfies Eq. (15), compute its Sampson error.

5. Repeat the above step many times (1000 times in our experiment) and choose the solution for which the Sampson error is the smallest.

\section{Experiment}

We generated four point sequences shown in Fig. 2 The points have equal arc length separations on each ellipse. Random Gaussian noise of mean 0 and standard deviation $\sigma$ is added independently to the $x$ and $y$ coordinates of each 


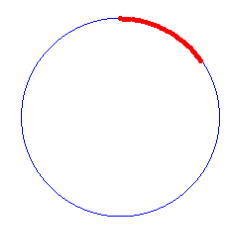

(a)

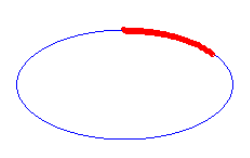

(b)

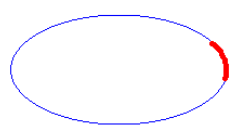

(c)

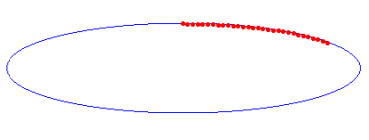

(d)

Fig. 2. Point sequences for our experiment. The number of points is $30,30,15$, and 30 and the average distance between neighboring points is $2.96,3.31,2.72$, and 5.72 for (a), (b), (c), and (d), respectively.

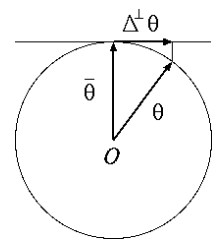

Fig. 3. The true value $\overline{\boldsymbol{\theta}}$, the computed value $\boldsymbol{\theta}$, and its orthogonal component $\Delta^{\perp} \boldsymbol{\theta}$ to $\overline{\boldsymbol{\theta}}$

point, and an ellipse is fitted. Since the computed value $\boldsymbol{\theta}$ and its true value $\overline{\boldsymbol{\theta}}$ are both unit vectors, we measure the error $\Delta \boldsymbol{\theta}$ by the orthogonal component 15

$$
\Delta^{\perp} \boldsymbol{\theta}=\boldsymbol{P}_{\bar{\theta}} \boldsymbol{\theta}
$$

where $\boldsymbol{P}_{\overline{\boldsymbol{\theta}}}\left(\equiv \boldsymbol{I}-\overline{\boldsymbol{\theta}} \overline{\boldsymbol{\theta}}^{\top}\right)$ is the orthogonal projection matrix along $\overline{\boldsymbol{\theta}}$ (Fig. 3). We evaluate the RMS error

$$
D=\sqrt{\frac{1}{10000} \sum_{a=1}^{10000}\left\|\Delta^{\perp} \boldsymbol{\theta}^{(a)}\right\|^{2}},
$$

over 10000 trials using different noise each time (the superscript $(a)$ indicates the value for the ath trial).

Figure 4 shows the ratio of non-ellipse occurrences by hyper-renormalization and the RMS error for the data in Fig. 2(a) (d). The horizontal axis indicates the noise level $\sigma$ divided by the average distance between neighboring points, which we call the relative noise level. Interrupted plots indicate that beyond that noise level convergence was not always reached after a specified number of iterations. The dotted lines indicate the KCR lower bound [810] given by

$$
D_{\mathrm{KCR}}=\frac{\sigma}{\sqrt{N}} \sqrt{\operatorname{tr} \overline{\boldsymbol{M}}_{5}^{-}}
$$


(a)
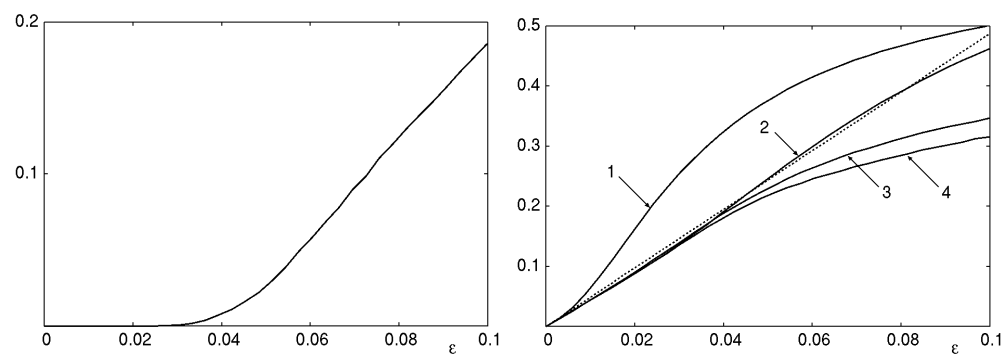

(b)
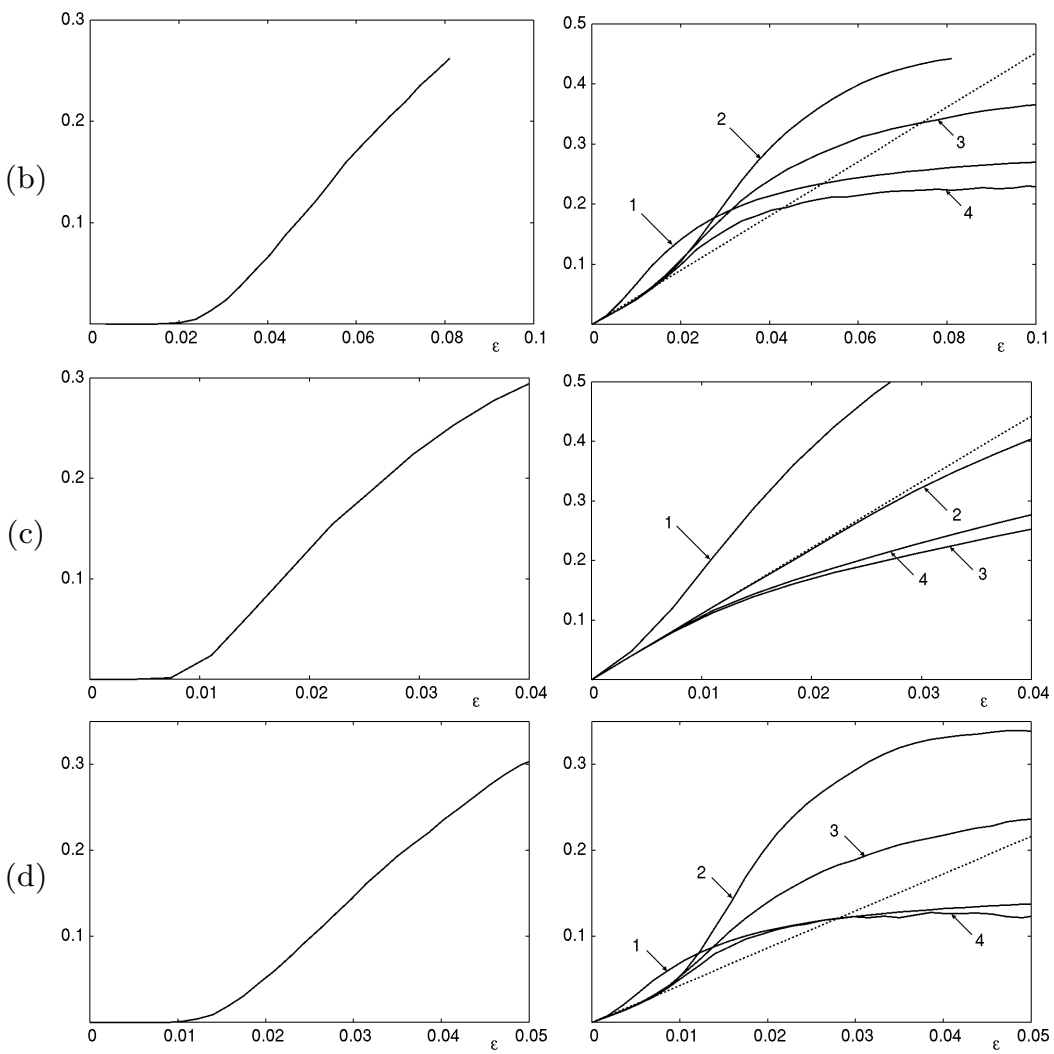

Fig. 4. Left column: The ratio of non-ellipse occurrences by hyper-renormalization for the data in Fig. 2(a) - (d). The horizontal axis is for the relative noise level $\epsilon$. Right column: The corresponding RMS fitting error: 1. The method of Fitzgibbon et al. 3 . 2. hyper-renormalization. 3. The method of Szpak et al. 23]. 4. Proposed method. The dotted lines indicate the KCR lower bound. Interrupted plots indicate that convergence is not reached after a specified number of iterations above that noise level. 


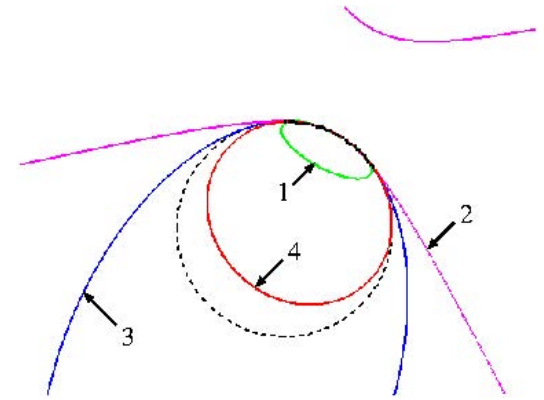

(a)

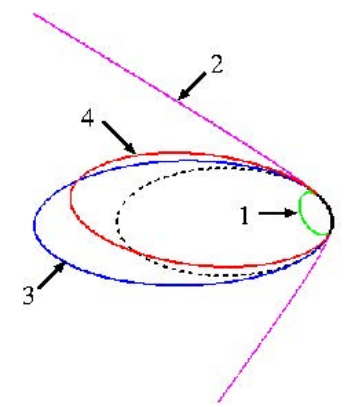

(c)

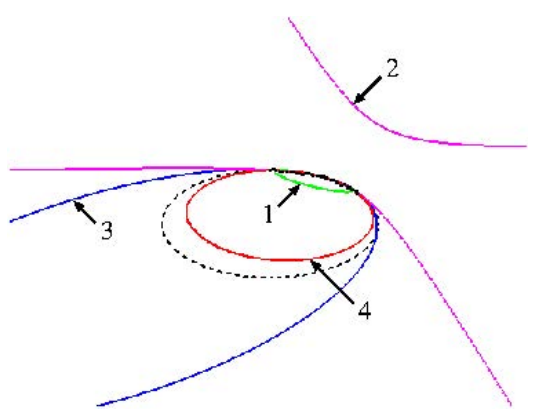

(b)

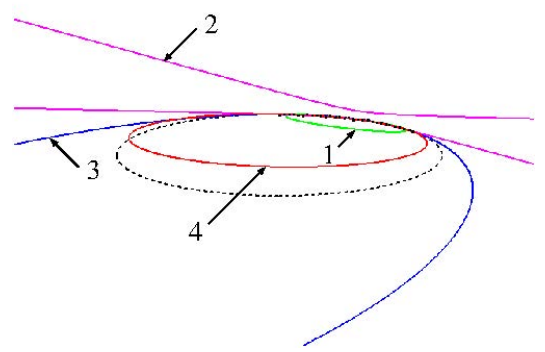

(d)

Fig. 5. Fitting examples for a particular noise when hyper-renormalization returns a hyperbola. 1. The method of Fitzgibbon et al. 3. 2. hyper-renormalization. 3. The method of Szpak et al. 23. 4. Proposed method. The dotted lines indicate the true shapes. The relative noise level $\epsilon$ is $0.169,0.151,0.092$, and 0.087 for (a), (b), (c), and (d), respectively.

As we can see, the accuracy of the method Fitzgibbon et al. [3] is generally very low, while hyper-renormalization 11 and the method of Szpak et al 223 are very accurate; they almost achieve the KCR lower bound when the noise is very small. However, the method Fitzgibbon et al. 3] can outperform hyperrenormalization and the method of Szpak et al. 23, when the points are chosen from a low-curvature part as in Fig. 2(d) and the noise is very large. Since the method of Szpak et al. 23] and the proposed method both restrict the solution to be an ellipse, their RMS error is smaller than hyper-renormalization in all cases, and in most cases our method is superior to that of Szpak et al. 23.

The reason that a hyperbola is fitted to an elliptic arc is, according to our interpretation, that a few heavily deviated points "pull" the curve to be a hyperbola. Such "bad points" are automatically ignored in the course of our random sampling, but all the points play the same role for the method of Szpak et al. 23. This may be the cause of higher performance of our method. However, we

\footnotetext{
${ }^{1}$ We used the code at: http://www.iim.cs.tut.ac.jp/ sugaya/

${ }^{2}$ We used the code at: https://sites.google.com/site/szpakz/
} 

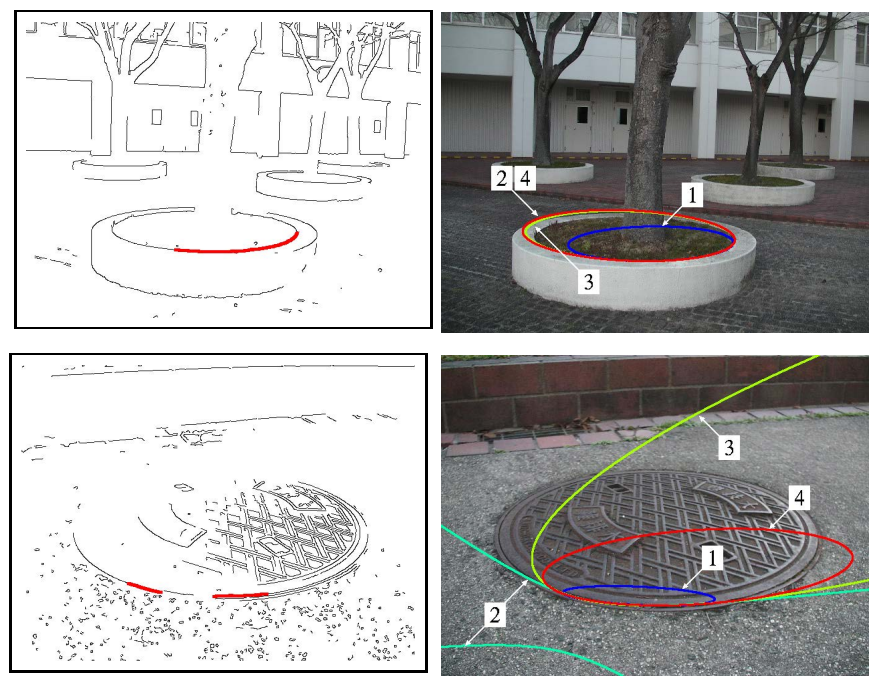

Fig. 6. Left: Edges extracted from real images. Red edge pixels (200 points above and 140 points below) are used for ellipse fitting. Right: Fitted ellipses superimposed on the original image. 1. The method of Fitzgibbon et al. 3]. 2. hyper-renormalization. 3. The method of Szpak et al. 23. 4. Proposed method.

observe that the difference between the method of Szpak et al. 223] and ours is very small for high-curvature arc as in Fig. 2(c).

Figure [5] shows fitting examples for a particular noise when hyperrenormalization returns a hyperbola. The dotted lines indicate the true shapes. We can observe a clear contrast: the method of Fitzgibbon et al. 3 fits a small and flat ellipse, while the method of Szpak et al. 23. fits a large ellipse close to the fitted hyperbola. Our method is in between, fitting an ellipse closer to the true shape.

The left of Fig. 6] shows edge images of real scenes. We fitted an ellipse to the edge points indicated in red (200 points above and 140 points below) by different methods. In the right, fitted ellipses superimposed on the original images are shown. The method of Fitzgibbon et al. 3. fits a small and flat ellipse in both cases. In the above example, hyper-renormalization returns an ellipse (hence our method) which is fairly accurate; the method of Szpak et al. 23] also fits an ellipse close to it. However, hyper-renormalization returns a hyperbola in the example below, and the method of Szpak et al. 23. fits a very large ellipse close to that hyperbola. We can see that our ellipse is somewhat between the small and flat ellipse of Fitzgibbon et al. 3] and the large ellipse of Szpak et al. 23.

\section{Concluding Remarks}

We have proposed a new method that always fits an ellipse to a point sequence extracted from images. The currently known best method is hyper-renormalization 
of Kanatani et al. 12, but it may return a hyperbola when the noise in the data is very large. Our proposed method incorporates random sampling so that an ellipse always results. Doing simulation, we showed that our method has higher accuracy than the method of Fitzgibbon et al. 3] and the method of Szpak et al. 23. the two currently known ellipse-specific methods. We also observed that when hyper-renormalization returns a hyperbola, the method of Szpak et al. [23] tends to fit a large ellipse close to that hyperbola while the method of Fitzgibbon et al. [3] tends to fit a small and flat ellipse. Our method fits an ellipse somewhat in between.

According to our experiences, we have never observed hyperbolas fitted to edge points of elliptic arcs in real applications unless artificial noise is added or edges are artificially limited to very short segments. If a hyperbola results from an elliptic arc, we should regard this as an indication of insufficient information in the data so that ellipse fitting no longer has meaning. Yet, even in such an extreme circumstance, our method can fit a tolerable ellipse.

Acknowledgements. The authors thank Wojciech Chojnacki of the University of Adelaide, Australia, and Ali Al-Shadraqah of the East Carolina University, USA for helpful discussions on ellipse fitting. This work was supported in part by JSPS Grant-in-Aid for Young Scientists (B 23700202) and for Challenging Exploratory Research (24650086).

\section{References}

1. Al-Sharadqah, A., Chernov, N.: A doubly optimal ellipse fit. Computational Statistics and Data Analysis 56(9), 2771-2781 (2012)

2. Chojnacki, W., Brooks, M.J., van den Hengel, A., Gawley, D.: On the fitting of surfaces to data with covariances. IEEE Trans. Patt. Anal. Mach. Intell. 22(11), 1294-1303 (2000)

3. Fitzgibbon, A., Pilu, M., Fisher, R.B.: Direct least squares fitting of ellipses. Patt. Anal. Mach. Intell. 21(5), 476-480 (1999)

4. Hartley, R., Zisserman, A.: Multiple View Geometry in Computer Vision, 2nd edn. Cambridge University Press, Cambridge (2004)

5. Ioannou, D., Huda, W., Laine, A.F.: Circle recognition through a 2D Hough transform and radius histogramming. Image Vis. Comput. 17(1), 15-26 (1999)

6. Kanatani, K.: Geometric Computation for Machine Vision. Oxford University Press, Oxford (1993)

7. Kanatani, K.: Renormalization for unbiased estimation. In: Pro. 4th Int. Conf. Comput. Vis (ICCV 1993), Berlin, Germany, pp. 599-606 (May 1993)

8. Kanatani, K.: Statistical Optimization for Geometric Computation: Theory and Practice. Elsevier, Amsterdam (1996); reprinted, Dover, York, NY, U.S.A. (2005)

9. Kanatani, K.: Ellipse fitting with hyperaccuracy. IEICE Trans. Inf. \& Syst. E89$\mathrm{D}(10), 2653-2660$ (2006)

10. Kanatani, K.: Statistical optimization for geometric fitting: Theoretical accuracy analysis and high order error analysis. Int. J. Comput. Vis. 80(2), 167-188 (2008)

11. Kanatani, K., Ohta, N.: Automatic detection of circular objects by ellipse growing. Int. J. Image Graphics 4(1), 35-50 (2004) 
12. Kanatani, K., Al-Sharadqah, A., Chernov, N., Sugaya, Y.: Renormalization Returns: Hyper-renormalization and Its Applications. In: Fitzgibbon, A., Lazebnik, S., Perona, P., Sato, Y., Schmid, C. (eds.) ECCV 2012, Part III. LNCS, vol. 7574, pp. 384-397. Springer, Heidelberg (2012)

13. Kanatani, K., Rangarajan, P.: Hyper least squares fitting of circles and ellipses. Comput. Stat. Data Anal. 55(6), 2197-2208 (2011)

14. Kanatani, K., Rangarajan, P., Sugaya, Y., Niitsuma, H.: HyperLS and its applications. IPSJ Trans. Comput. Vis. Appl. 3, 80-94 (2011)

15. Kanatani, K., Sugaya, Y.: Performance evaluation of iterative geometric fitting algorithms. Comput. Stat. Data Anal. 52(2), 1208-1222 (2007)

16. Kanatani, K., Sugaya, Y.: Unified computation of strict maximum likelihood for geometric fitting. J. Math. Imaging Vis. 38(1), 1-13 (2010)

17. Kanatani, K., Sugaya, Y.: Hyperaccurate correction of maximum likelihood for geometric estimation. IPSJ Trans. Comput. Vis. Appl. 5, 19-29 (2013)

18. Leedan, Y., Meer, P.: Heteroscedastic regression in computer vision: Problems with bilinear constraint. Int. J. Comput. Vis. 37(2), 127-150 (2000)

19. Matei, J., Meer, P.: Estimation of nonlinear errors-in-variables models for computer vision applications. IEEE Trans. Patt. Anal. Mach. Intell. 28(10), 1537-1552 (2006)

20. Roth, G., Levine, M.D.: Extracting geometric primitives. CVGIP: Image Understand 58(1), 1-22 (1993)

21. Roth, G., Levine, M.D.: Geometric primitive extraction using a genetic algorithm. IEEE Trans. Pattern Anal. Mach. Intell. 16(9), 901-905 (1994)

22. Rosin, P.L., West, G.A.W.: Nonparametric segmentation of curves into various representations. IEEE Trans. Pattern Anal. Mach. Intell. 17(12), 1140-1153 (1995)

23. Szpak, Z.L., Chojnacki, W., van den Hengel, A.: Guaranteed ellipse fitting with Sampson distance. In: Fitzgibbon, A., Lazebnik, S., Perona, P., Sato, Y., Schmid, C. (eds.) ECCV 2012, Part V. LNCS, vol. 7576, pp. 87-100. Springer, Heidelberg (2012)

24. Taubin, G.: Estimation of planar curves, surfaces, and non-planar space curves defined by implicit equations with applications to edge and range image segmentation. IEEE Trans. Patt. Anal. Mach. Intell. 13(11), 1115-1138 (1991) 